\title{
Placa de protección mucosa, elemento de uso actual en el desafío de la protección de la mucosa oral
}

\section{Mucosa protection plate, current use element in the challenge of the protection of oral mucosa}

\author{
Eliana Guzmán-Menco ${ }^{1 \oplus}$, Martha Carmona-Lorduy ${ }^{2}{ }^{(}$, Jonathan Harris-Ricardo $^{3(0)}$
}

Tipología: Reporte de caso clínico.

Para citar este artículo: Guzmán-Menco E, Carmona-Lorduy M, Harris-Ricardo J. Placa de protección mucosa, elemento de uso actual en el desafío de la protección de la mucosa oral. Duazary. 2019 septiembre; 16(3): 112 - 120. Doi: http://dx.doi.org/10.21676/2389783X.2974

Recibido en febrero 01 de 2018

Aceptado en marzo 22 de 2019

Publicado en línea en septiembre 01 de 2019

\section{RESUMEN}

La placa de protección mucosa (PPM) es un aparato o dispositivo flexible que disminuye la incidencia y severidad del trauma en el tejido blando bucal, preservando así la integridad de este. Es utilizada como alternativa terapéutica en lesiones de cavidad oral asociadas a trauma, y además de forma preventiva en aquellos pacientes con antecedentes de algunos hábitos como morsicatio buccarum, entre otros. Se reportan dos casos remitidos al posgrado de Estomatología y Cirugía Oral de la Universidad de Cartagena, Colombia. Estos pacientes presentaron lesiones traumáticas en diferentes sitios de la mucosa bucal, para lo cual se implementó el uso de una placa de protección mucosa con el objetivo de aislar el trauma crónico localizado sobre estos, lo cual permitió obtener finalmente resultados positivos en corto tiempo.

Palabras clave: mucosa bucal; prevención; deporte; protector bucal.

1. Universidad de Cartagena. Cartagena, Colombia. Correo: eliaguzman04@ hotmail.com - https://orcid.org/0000-0002-8475-6301

2. Universidad de Cartagena. Cartagena, Colombia. Correo: mcarmonal@ unicartagena.edu.co - https://orcid.org/0000-0003-3066-2219

3. Universidad de Cartagena. Cartagena, Colombia. Corporación Universitaria Rafael Núñez. Cartagena, Colombia. Correo:

j.harris.r@hotmail.com - https://orcid.org/0000-000246620729 


\section{ABSTRACT}

The mucosal protection plaque (MPP) is a flexible device, which decreases the incidence and severity of trauma in the soft tissue of the mouth, is used as a therapeutic alternative in oral cavity lesions, associated with trauma, and also in a preventive way in those patients with a history of some habits such as morsicatio buccarum among others, mainly this device seeks to preserve the integrity of soft tissues. Two cases referred to the postgraduate course of Stomatology and Oral Surgery of the Cartagena's University, Colombia, are reported. These patients presented traumatic lesions in different sites of the oral mucosa, for which the use of a mucosal protection plaque was implemented, in order to isolate the localized chronic trauma, finally obtaining positive results in a short time.

Keywords: buccal mucosa; prevention; sport; mouthguard.

\section{INTRODUCCIÓN}

Las lesiones traumáticas de la mucosa oral constituyen un grupo de alteraciones que afectan el funcionamiento de la cavidad bucal. La agresión producida en esta mucosa puede ser ocasionada por diversos factores traumáticos como dientes con bordes filosos o cortantes, aparatología protésica u ortodóntica, hábitos como el de queilofagia, deportes extremos, llevar elementos extraños a la cavidad oral como palillos y bolígrafos, entre otros ${ }^{1,2}$.

Estas lesiones pueden presentar sintomatología leve a moderada, lo que muchas veces puede causar una descompensación en el paciente, pues se ven limitadas la masticación y la deglución, específicamente cuando se ubican en la lengua. Además, se ha comprobado que un irritante crónico localizado puede ser el desencadenante de desórdenes potencialmente malignos ${ }^{3}$.

Existen métodos preventivos cuyo objetivo es la protección de los dientes y mucosa oral frente a traumas contundentes, entre los cuales se encuentran el protector bucal para deportes de alto impacto y otros que se utilizan con mayor frecuencia como alternativa terapéutica en las lesiones relacionadas con trauma, como la placa de protección mucosa $(\mathrm{PPM})^{4}$. Esta es un dispositivo flexible y suave que reduce la incidencia y severidad del trauma en el tejido blando bucal, brindándoles protección a la mucosa y a la lengua, aislando los tejidos del traumatismo constante causante de la lesión. Esta placa es removible, debe permanecer el mayor tiempo posible en boca y se retira para realizar higiene oral y alimentación. El paciente puede dormir con la placa, excepto si presenta bruxismo nocturno; en este caso debe retirarla antes de dormir, ya que es posible que las fuerzas oclusales alteren la morfología del material. Además, es importante eliminar el factor etiológico para evitar la recidiva ${ }^{5,6}$.

El uso de la PPM está indicado en lesiones de origen traumático que afecten tejido blando y en pacientes que presenten hábitos autolesivos o aparatología ortodóntica. El material utilizado en la confección de la placa es polímero termoplástico conformado por unidades de etileno y acetato de vinilo, de consistencia blanda. Esta última particularidad del material es importante, ya que las placas rígidas podrían generar lesiones en el tejido blando ${ }^{3-7,8}$. Asimismo, otras características que debe presentar la PPM son: permitir la deglución y la fonación; que, al ser lavada y desinfectada, no se deforme; garantizar que la retención esté proporcionada por la arcada dentaria; y no ser tóxica $^{7-9}$.

Antes de elaborar la PPM se debe realizar diagnóstico de la lesión y asegurarse de que su causa es de origen traumático; además, es preciso realizar fases higiénicas para disminuir la carga bacteriana en cavidad oral. También se requiere eliminar los bordes contantes de los dientes que los presenten y realizar restauración en los dientes necesarios. Luego se toma una impresión en alginato para hacer el vaciado en 
yeso piedra, y de esta manera se obtiene el modelo sobre el cual se va a realizar la PPM. Los límites de la placa se dibujan en dicho modelo, y este se coloca después sobre la parrilla de la máquina Vacuum. La lámina de vinilo se inserta en el anillo y se sube hacia lo más próximo a la resistencia. Cuando esta última se activa, el calor generado por ella va reblandeciendo la lámina, formándose así una gota que debe tener aproximadamente un pulgada. Es aquí cuando se apaga la resistencia y se baja el anillo que sujeta la lámina sobre el modelo para luego activar el succionador o aspiradora durante 40 segundos. Cuando se ha enfriado a temperatura ambiente, se realiza el recorte de la lámina, con tijeras o con mango de bisturí número tres y hoja número 15. El recorte debe seguir la línea que se demarcó inicialmente sobre el modelo ${ }^{3-9}$.

La PPM amerita cuidados especiales durante su utilización tales como limpiarla con un cepillo de cerdas suaves, enjuagarla con una solución antiséptica para evitar la colonización de microorganismos, y no lavarla o sumergirla en agua caliente para que no se deforme ${ }^{3,4-10}$.

El propósito del presente reporte de casos es mostrar los resultados clínicos terapéuticos obtenidos con la utilización de la PPM en pacientes que acudieron a la Unidad Estomatológica de la Facultad de Odontología de la Universidad de Cartagena.

\section{REPORTE CASO UNO}

Paciente de 13 años de edad que presentó úlcera en mucosa labial superior, con tiempo de evolución de 15 días. El paciente reportó antecedentes de neuropatía y síndrome de Tourette. Según comentó su familia, desde hacía un año la separación de los padres y las constantes discusiones generaban episodios de estrés en el paciente.

En el examen físico se observaron movimientos inconscientes; fueron notorias las expresiones orales involuntarias y la coprolalia características del síndrome. Intraoralmente, se observó una úlcera en mucosa labial superior, lado derecho, de aproximadamente $2 \mathrm{~cm}$ de diámetro, fondo blanquecino, bordes crateriformes, moderadamente sintomática a la palpación (Figura 1). El paciente negó el uso de analgésicos para el control de sintomatología dolorosa. Se realizó la impresión clínica de úlcera asociada a traumatismo crónico.

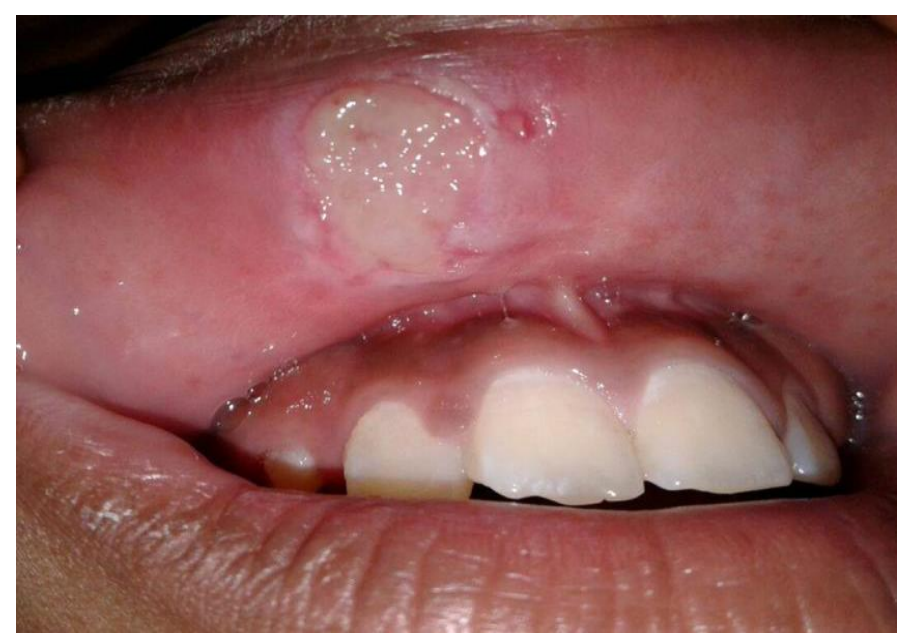

Figura 1. Úlcera en mucosa labial superior 
Como terapéutica se decidió utilizar la placa de protección mucosa en el maxilar, con placa de acetato número 0,2 (Figura 2). Se le indicó al paciente que solo debía retirarse la placa en el momento de la masticación y deglución de alimentos y se programaron citas de control cada ocho días para observar el proceso de cicatrización de la lesión. Luego de 30 días de la utilización de la PPM, se observó disminución del tamaño de la úlcera, y en el control a los a los 45 días (Figura 3) se observó cicatrización completa de la lesión. Además, se instauraron terapias psicológicas para el tratamiento de la enfermedad de base.

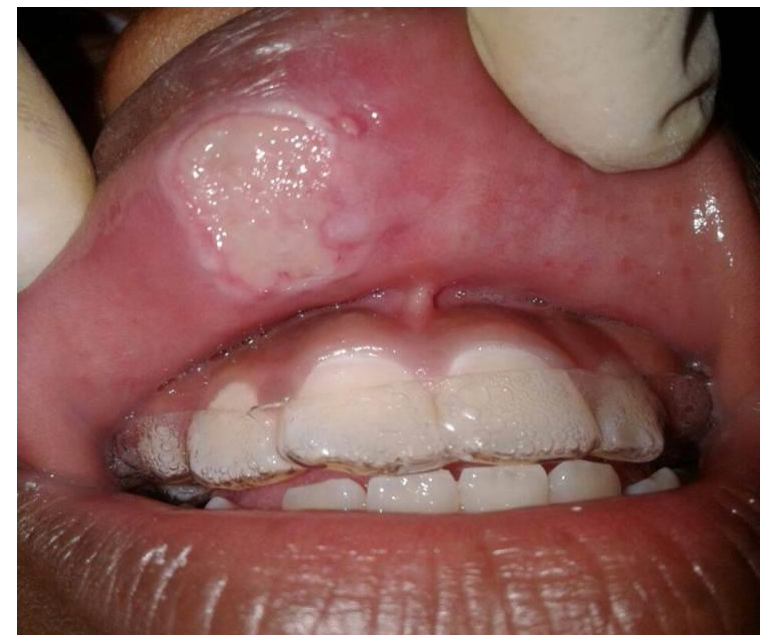

Figura 2. Utilización de la placa de protección mucosa

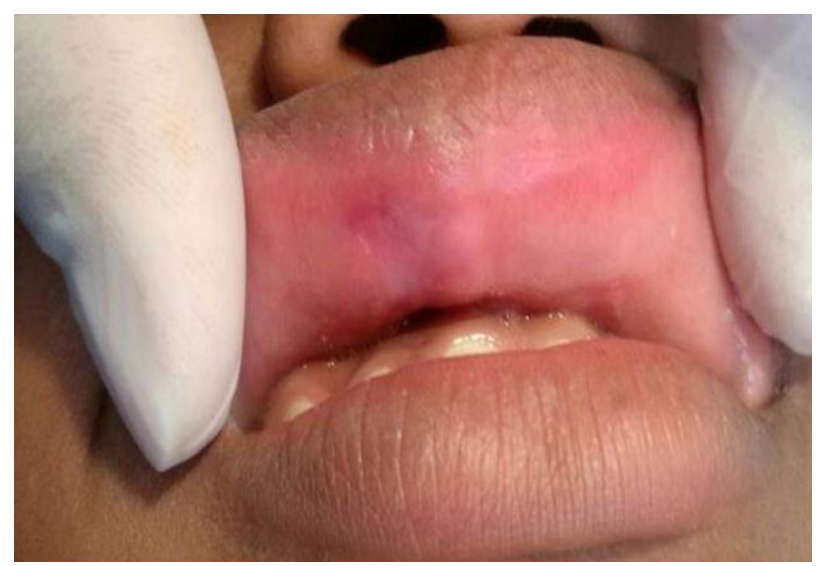

Figura 3. Control realizado a los a los 45 días, donde se observó cicatrización completa de la lesión 


\section{REPORTE CASO DOS}

Paciente de 22 años de edad, remitida por presentar múltiples lesiones asociadas al hábito de mordisqueo y localizadas en mucosa yugal bilateral (Figuras 4a, 4b), con tiempo de evolución de cinco años aproximadamente. No presentó antecedentes familiares ni personales relevantes para el caso.

En el examen clínico intrabucal se observaron múltiples colgajos epiteliales con indentaciones en mucosa yugal, bilateral, en toda su extensión. Además, se registraron lesiones ulcerativas de diversas formas y que oscilaban entre $5 \mathrm{~mm}$ y 1 $\mathrm{cm}$ de longitud, sintomáticas y áreas sangrantes con placa blanquecina, asintomática, asociadas al hábito de mordisqueo, el cual aumentaba cuando la paciente era sometida a episodios de estrés, con impresión clínica de mucosa mordisqueada o morsicatio buccarum e hiperqueratosis friccional.

4B

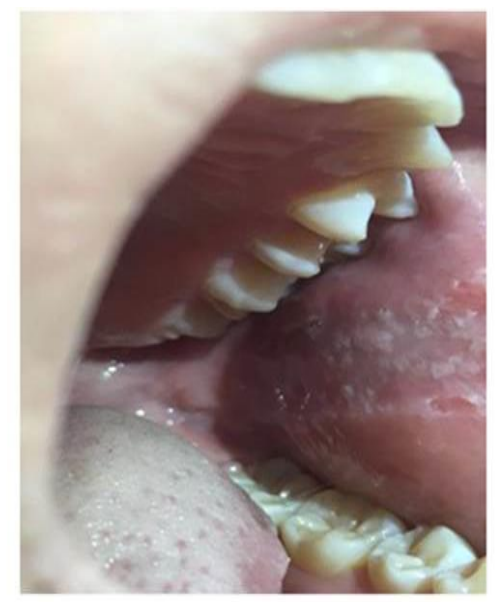

Figura 4a y 4b. Múltiples lesiones traumáticas asociadas al hábito de mordisqueo

Para el tratamiento se realizó la PPM en el maxilar superior con placa de acetato número 0,2 (Figura 5), con indicaciones de retirarla solo en el momento de la alimentación, y se programó cita de control a los ocho días. Para entonces solo se observaron placas en mucosa yugal de los lados izquierdo y derecho de aproximadamente
$4 \mathrm{~mm}$, un tamaño menor al notado en el examen intrabucal inicial, lo que confirmó signos indicativos de buen proceso de cicatrización. En el control realizado a los 23 días, se observó resolución completa de las lesiones (Figura 6a, $6 b)$. 
Placa de protección mucosa, elemento de uso actual en el desafío de la protección de la mucosa oral

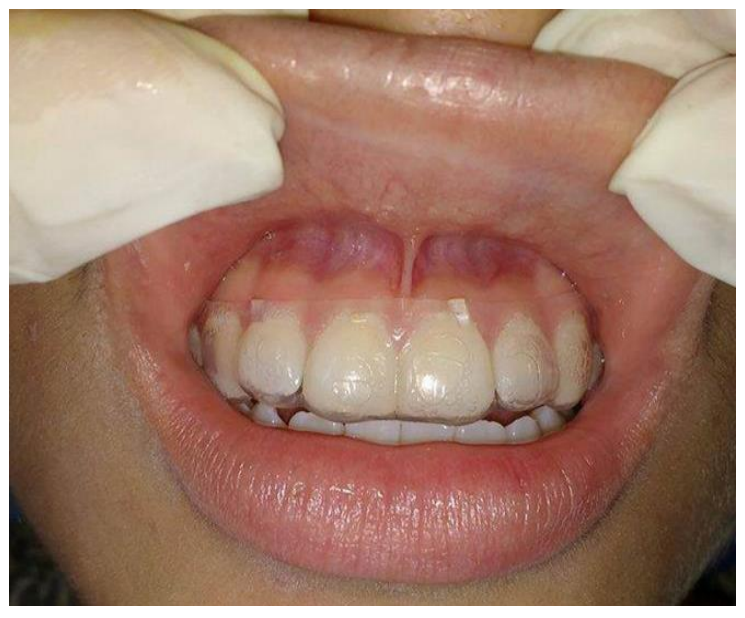

Figura 5. Placa de protección mucosa
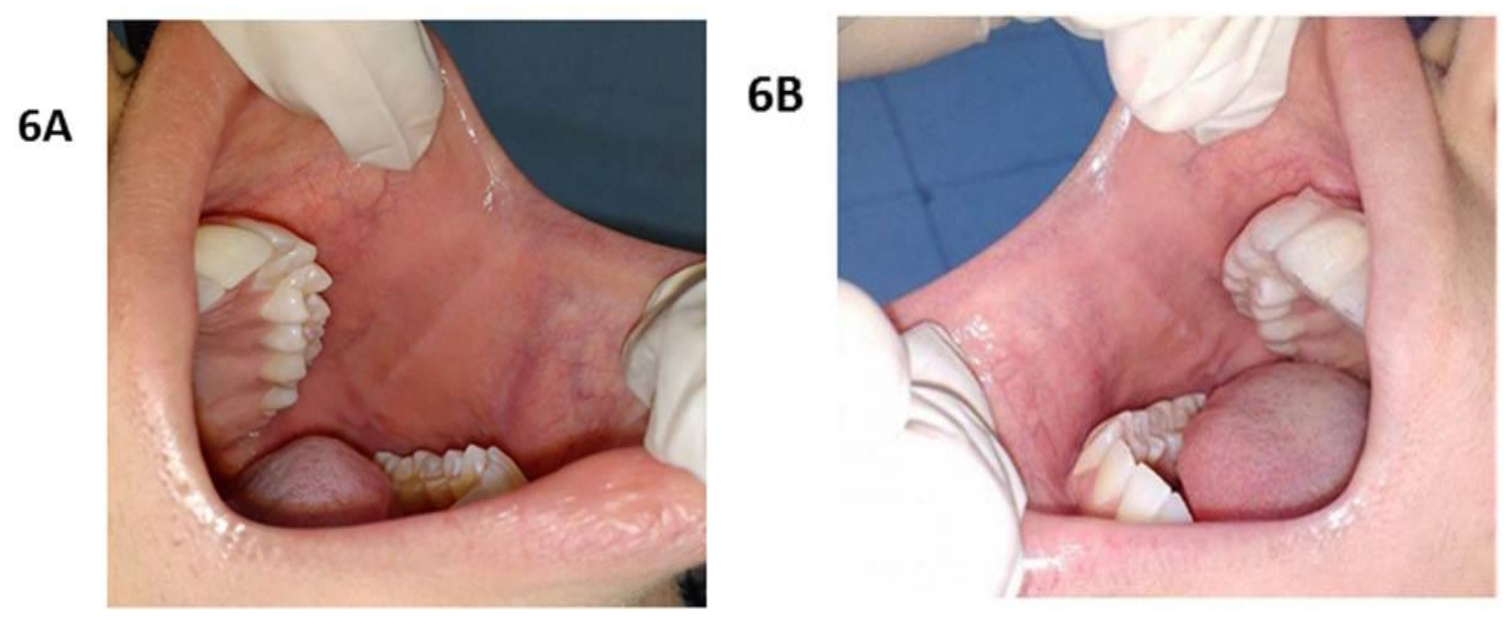

Figura 6a y 6b. Control realizado a los 23 días, en el que se observó resolución completa de las lesiones en mucosa yugal bilateral

\section{DISCUSIÓN}

La PPM es un dispositivo flexible que reduce la incidencia y severidad del trauma en el tejido blando bucal, brindándoles protección a la mucosa y a la lengua, aislando los tejidos del roce o traumatismo constante causante de la lesión ${ }^{4}$.

De la Teja et $a l .^{6}$ mencionan que en México realizaron un estudio en el que evaluaron los resultados de los dispositivos intraorales como guardas o placas de protección bucal y férulas para pacientes pediátricos. Los resultados destacaron que las placas de protección simple o PPM de acetato flexible son útiles para prevenir lesiones traumáticas en tejidos blandos como lengua, encía y mucosas. Asimismo, se afirmó que estos dispositivos son capaces de mejorar el cuadro clínico de lesiones traumáticas 
intraorales establecidas, lo que concuerda con la presente serie de casos, en la que se utilizó la PPM en tres pacientes con ese tipo de heridas que afectaron la mucosa oral y se obtuvieron resultados positivos.

Yamanaka et al. $^{11}$ comentan que en Tokio reportaron el manejo de un paciente con la lengua lacerada por un diente con bordes cortantes después de una crisis convulsiva. En este caso utilizaron un protector bucal para impedir que el diente entrara en contacto con la lengua, y de esta manera se protegió la lengua del trauma constante. La lesión cicatrizó gradualmente hasta su desaparición, lo cual, de nuevo, coincide con los casos anteriormente reportados ya que la PPM, además de proteger los tejidos blandos, ayudó en la cicatrización de las lesiones en la mucosa, impidiendo el traumatismo de esta.

Das et $a l .{ }^{12}$ recomiendan, después de una laceración en la parte anterior de la lengua, la fabricación de una placa de protección con superficie lisa. Esto es importante, según los autores, para la prevención de la irritación de la herida por los dientes y evitar que se afecte el proceso de cicatrización. Tal hallazgo concuerda con el actual reporte de pacientes con lesiones en la mucosa bucal, en que la PPM actuó como un medio aislante que impidió el contacto directo de los dientes con las lesiones y arrojó buenos resultados.

Rivera et al. ${ }^{13}$ indican que en México colocaron un protector bucal en un adulto para evitar la automutilación de los tejidos blandos de la cavidad bucal debido a antecedentes de un infarto en tallo cerebral mesencefálico que generó movimientos involuntarios en la boca, presentando desgaste agresivo de los dientes. Los autores reportaron que los resultados fueron satisfactorios y destacaron que el material ideal para la realización del protector bucal debe ser blando, flexible y delgado para no interferir con la oclusión, propiedades que brinda el etileno de acetato de vinilo ${ }^{14,15}$. Esto coincidie con el presente reporte, en el que se utilizó etileno de acetato de vinilo como material ideal para la elaboración de la PPM.

Para finalizar, se afirma que la PPM es la actual alternativa preventiva y terapéutica utilizada en lesiones de la boca debido a los resultados clínicos terapéuticos obtenidos con la utilización del elemento frente a dichas heridas.

\section{DECLARACIÓN SOBRE CONFLICTO DE INTERESES}

Los autores declaran no conflicto de intereses y no financiación.

\section{CONTRIBUCIÓN DE LOS AUTORES}

Primer autor: revisión de la literatura, elaboración del manuscrito, realización de la PPM en los laboratorios y controles periódicos de los pacientes con las PPM.

Segundo autor: dirección del proyecto, elaboración del manuscrito, diagnóstico de los casos y elección de la terapéutica para los casos clínicos, diseño de la placa de la protección mucosa y redacción.

Tercer autor: revisión de la literatura, elaboración del manuscrito.

\section{REFERENCIAS BIBLIOGRÁFICAS}

1. Somacarrera M, López A, Martín C, Díaz M. Lesiones traumáticas en la mucosa oral de los adultos mayores. Av Odontoestomatol. 2015;31(3):129-134. Doi: http://dx.doi.org/10.4321/S021312852015000300003

2. Corral C, Mercado M, Latapiat A, Veliz A, Garrido M. Protector bucal por laminado a presión en paciente deportista. Rev Clin Periodoncia Implanmtol Rehabil Oral. 2017;10(3);166-168.

Doi: http://dx.doi.org/10.4067/S071901072017000300166 
Placa de protección mucosa, elemento de uso actual en el desafío de la protección de la mucosa oral

3. Álvarez E, Duque C. Lesiones bucales potencialmente malignas. Rev Acta de Otorrinolaringología \& Cirugía de Cabeza y Cuello. 2013;41(1):51-56 [citado 2018 Jun 08]. Disponible en: https://www.acorl.org.co/articulos/14060312 1814.pdf

4. Parker K, Marlow B, Patel N, Gill DS. A review of mouthguards: effectiveness, types, characteristics and indications for use. $\mathrm{Br}$ Dent J. 2017;222(8):629-633. Doi: 10.1038/sj.bdj.2017.365

5. Silveira EG, Araújo SM, Schmitt BHE, Farías MMAG, Campos L, Caregnato M. Conhecimento e atitudes dos odontopediatras do Estado de Santa Catarina acerca de mecanismos de prevencao de traumatismos bucais relacionados a esportes. Rev Odontol UNESP. 2009;38(6):341-6 [citado 2018 Feb 03]. Disponible en: http://s3.amazonaws.com/host-articleassets/rou/588018a87f8c9d0a098b4d60/fullt ext.pdf

6. De la Teja-Ángeles E, Zurita Bautista YE, Durán A. Dispositivos intraorales: guardas oclusales y férulas para pacientes en estado crítico. Experiencia del Instituto Nacional de Pediatría. Acta Pediatr Mex 2012;33(4):19197 [citado 2017 Nov 20]. Disponible en: http://www.medigraphic.com/pdfs/actpedme x/apm-2012/apm124f.pdf

7. Ranalli D. Prevention of sports-traumatic dental injuries. Dent Clin North Am. 2000;44:35-51 [citado 03 jul 2018]. Disponible en: https://www.ncbi.nlm.nih.gov/pubmed/1063 5467

8. Guinot F, Torrents R, Armengol A, Cuadros $\mathrm{C}$, Lorente A. Protector bucal individualizado mediante la técnica de formación al vacío. A propósito de un caso. Odontol Pediátr (Madrid). 2014;22(3):195-208 [citado 2018
Abr 02]. Disponible en: https://www.odontologiapediatrica.com/wpcontent/uploads/2018/08/268_04_OR_279_J imeno.pdf

9. Knapik JJ, Marshall SW, Lee RB, Darakjy SS, Jones SB, Mitchener TA. Mouthguards in sport activities: history, physical properties and injury prevention effectiveness. Sports Med Auckl NZ. 2007;37(2):117-44. Doi: 10.2165/00007256-200737020-00003

10. Escarrá V, Fernández M, Machado R, Cabrera A. Necesidad del uso de protectores bucales en niños que practican deportes de bajo y mediano riesgo. Valencia, noviembre 2013 [Trabajo de grado]. Universidad de Carabobo, Facultad de Odontología, Dpto. de Formación Integral del Hombre Informe de Investigación. [Citado 2018 Ene 08]. Disponible en: http://mriuc.bc.uc.edu.ve/bitstream/handle/1 23456789/2881/Vermar.pdf?sequence $=1$

11. Yamanaka R, Soga Y, Moriya Y, Okui A, Takeuchi T, et al. Management of lacerated and swollen tongue after convulsive seizure with a mouth protector: interprofessional collaboration including dentists in intensive care. Acta Med Okayama. 2014;68(6):375-8. Doi: 10.18926 / AMO / 53027

12. Das UM, Gadicherla P. Lacerated tongue injury in children. Int $\mathrm{J}$ Clin Pediatr Dent. 2008;1(1):39-41 [citado 2018 Ag 27]. Disponible en: https://www.ncbi.nlm.nih.gov/pmc/articles/P MC4086539/

13. Rivera M, Pérez J, Marín O. Aplicación de un protector bucal hecho a la medida para evitar la automutilación. Rev Nal Odontol Méx. 2010;2(12):6-12 [citado 2018 Jul 15]. Disponible en: http://imbiomed.com/1/1/articulos.php?meth od=showDetail\&id_articulo=67081\&id_secc ion $=3880 \&$ id_ejemplar $=6742 \&$ id_revista $=2$ 35 
14. Mancebo E. Técnicas de laboratorio para la confección de protectores bucales bilaminados en pacientes portadores de aparatología ortodóncica fija. Rev Odontol. 2005;11(23-28) [citado 2018 Ag 01]. Disponible en: https://es.scribd.com/document/99435599/Pr otector-Bucal
15. Duddy FA, Weissman J, Lee RA, Paranjpe A, Johnson JD, Cohenca N. Influence of different types of mouthguards on strength and performance of collegiate athletes: a controlled-randomized trial: Mouthguards and athletic strength and performance. Dent Traumatol. 2012 agosto;28(4):263-7. Doi: 10.1111 / j.1600-9657.2011.01106.6.x 